\title{
The role of mitochondria in vascular calcification
}

Pengbo Wang, Naijin Zhang, Boquan Wu, Shaojun Wu, Ying Zhang, Yingxian Sun

Department of Cardiology, The First Hospital of China Medical University, Shenyang, Liaoning Province, China

\section{ABSTRACT}

Vascular calcification (VC) was defined as the ectopic deposition of calcium-phosphorus complexes on the blood vessel walls. It was a process involving multiple factors and mechanisms, covering the phenotype transition of vascular smooth muscle cells (VSMCs) and release of microvesicles. It was a common end-stage alteration of chronic diseases such as cardiovascular disease and chronic kidney disease. Increasing evidence indicates that mitochondria were involved in the development of VC. Mitochondria provided energy to cells, maintained the stability of cell functions, and participated in a variety of biological behavior. Oxidative stress, autophagy, apoptosis, and mitochondrial DNA (mtDNA) damage could affect the development of VSMCs calcification by alteration of mitochondrial function. This article reviewed the mechanism of calcification and the role of mitochondria in VC, aiming to raise a novel insight into drug development and clinical treatment.

Key words: vascular calcification, mitochondria, oxidative stress, autophagy, apoptosis, mitochondrial DNA

\section{INTRODUCTION}

Vascular calcification (VC) was a pathological phenomenon that calcium and phosphorus were deposited on the vessel wall in the form of hydroxyapatite; it was commonly found in the intimal or media artery. ${ }^{[1]}$ The arterial intima calcification was generally related to atherosclerotic plaques, and the calcification in the media of vascular was a frequent end-stage pathological change of most chronic diseases, especially happened in hypertension, diabetes mellitus, and chronic kidney diseases. ${ }^{[2,3]}$ According to some epidemiological studies, VC was found in $80 \%$ and $90 \%$ of patients with vascular injury and coronary heart diseases, respectively, ${ }^{[4]}$ besides, the presence of calcification usually predicted poor clinical outcomes especially in coronary atherosclerotic diseases. ${ }^{[5]}$ Mitochondria were the most crucial energy metabolism factories of cells. Mitochondria could produce adenosine triphosphate (ATP) and a variety of biosynthetic intermediates, participate in oxidative stress, and affect autophagy and apoptosis. Therefore, the quality and activity of mitochondria were closely related to the state of cells. A variety of mechanisms participate in the deposition of calcium in the blood vessel walls, and mitochondria played an essential role in the process of VC. In the recent years, the impact of mitochondria on calcification has received increasing attention. This review has summarized the role of mitochondria in calcification from mitochondrial oxidative stress damage, regulation of autophagy and apoptosis, and mitochondrial DNA (mtDNA) damage.

\section{VASCULAR CALCIFICATION}

\section{Mechanism of VC}

Increasing evidence indicated that the process of VC had a similar program with bone formation, and $\mathrm{VC}$ was an active and highly regulated process based on the phenotype transition of vascular smooth muscle cells (VSMCs). ${ }^{[6]}$ The whole mechanism of calcification was still unclear, but there were two main characteristics of VSMCs calcification, the transition of VSMCs into osteogenic phenotype and the secretion of extracellular vesicles (EVs). 
Usually, VSMCs were in a contractile phenotypic state and played an essential role in keeping a stable contractile function of VSMCs. Under the situations of various stimulating factors, especially oxidative stress damage, abnormally accumulated reactive oxygen species (ROS) and calcium-phosphorus imbalance would upregulate the expression of osteoblast-promoting factor Runtrelated transcription factor 2 (Runx) and promote the differentiation of VSMCs to the osteogenic phenotype. ${ }^{[7,8]}$ And the other stimuli such as senescence, chronic inflammation, and even exosomes contained micro-RNA, could change VSMCs into the osteogenic phenotype; during this process, the specific gene expression of the smooth muscle contraction phenotype, such as smooth muscle $22-\alpha$ protein (SM22 $\alpha$ ), alpha-smooth muscle actin $(\alpha$-SMA), and smooth muscle myosin-heavy chain (SMM$\mathrm{HC}$ ), was decreased. In contrast, the expression of core transcription factors, such as co-rebinding factor alpha 1 (Cbf $\alpha 1)$, muscle segment homeobox 2 (MSX2), alkaline phosphatase (ALP), and other osteogenic genes, were upregulated, a variety of calcification promoting factors and inhibitors were imbalanced, and the expression of a large number of bone matrix proteins provided a cellular basis for the formation of calcifications. ${ }^{[9-11]}$

The main component of EVs was calcium phosphate crystals. Calcium phosphate minerals precipitated when sufficient $\mathrm{Ca}^{2+}$ and $\mathrm{PO}_{4}^{3-}$ have accumulated within EVs. ${ }^{[12]}$ The secretion of EVs not only provided a material basis for the calcification process but also further stimulated the cells to undergo osteogenic differentiation. The high calcium level in the cytoplasm would promote the translocation of Annexin-6 and transport to reposition in the plasma membrane, thereby triggering the release of vesicles, which would serve as a platform or calcified core outside the cell for calcium and phosphorus deposition and further re-combines and stimulates the epitaxial growth of hydroxyapatite, and the sediment would further stimulate cells to undergo apoptosis. ${ }^{[4,13,14]}$ In addition, the matrix vesicles (MVs), vesiclelike apoptotic bodies formed by the apoptotic reaction, could also enrich calcium. ${ }^{[10]}$ At the same time, the apoptosis of cells would accelerate the triggering of the release of EVs. ${ }^{[15]}$

These two processes were causality relationships, but could also affect each other, eventually would lead to $\mathrm{VC}$, and the calcification further aggravated the process of VSMCs phenotype transition. ${ }^{[16-18]}$ Besides the above two main processes, there were several other factors or diseases that could affect calcification by regulating the microenvironment of VSMCs, such as the shearforce of blood flow on the vessel wall, blood glucose or lipid levels, and calcium-phosphorus imbalance, ${ }^{[5,19,20]}$ especially hypertension had the most visible effects on $\mathrm{VC}$ microenvironment alteration.
Research has reported that Runx2 expression levels were significantly upregulated in pulmonary VSMCs of patients with pulmonary hypertension, and the location of Runx2 expression had a significant correlation with the site of pulmonary calcification. ${ }^{[21]}$ In the rat model of VC, it was found that the increase in intravascular calcium deposition was closely related to the increased angiotensin II and aldosterone levels, meanwhile the VC status could be inhibited by captopril or spironolactone, ${ }^{[22]}$ furthermore in the renal artery vascular also presented a massive calcium deposition status. ${ }^{[23,24]}$ Moreover, in the rat model that showed growth-related spontaneous hypertension, VSMCs underwent a transition into a chondroblast phenotype. ${ }^{[25]}$ These studies suggested that hypertension-induced vascular remodeling, such as changes in the extracellular matrix, could make the VSMCs microenvironment conducive to calcium deposition and subsequently form $\mathrm{VC}$ under other harmful stimuli or promote calcification factors.

We have already known that the extent of calcium and phosphorus deposition depends on the concentration of $\mathrm{Ca}^{2+}$ and phosphate. ${ }^{[2]}$ The phenotype transition and VC induced by high phosphate or high calcium were depended on phosphate transporter traffic facilitator-1 (Pit-1). High level of calcium or phosphate could upregulate and activate Pit-1. ${ }^{[27]}$ The activation of Pit-1 could increase the intake of inorganic phosphate (Pi), and high level of intracellular phosphorus would lead to the phenotype transition of VSMCs and regulate the extracellular matrix that was conducive to VC. ${ }^{[28]}$ On the other hand, the activation of Pit-1 could also increase the Pi in EVs and promote the EVs release, ${ }^{[29]}$ high level of calcium could enhance the effects of Pit-1 on Pi intake, ${ }^{[27]}$ thereby promoting the formation of vesicle calcification cores. ${ }^{[30]}$

\section{'Soil-Seed-Pest' mechanism model}

VC was a complex, multifactor, multiprocess and can be a regulated process. Each component in the calcification process affected and interacts with each other. Taken together, based on the above processes, we propose a "SoilSeed-Pest" model to describe the process of VC (Fig. 1). Alteration and imbalance of VSMCs microenvironment and vascular environment (soil); damage to VSMCs (seed) exposed to calcification environment, especially VSMCs which had transformed into osteogenic phenotype and senescence VSMCs; and various extracellular stimuli (pest) such as ROS damage, inflammation, and hypertension are the three elements that worked together and eventually lead to the outcome of VC.

In the early stage of VC, VSMCs microenvironment took place abnormal alterations, such as calcium-phosphorus imbalance, gave rise to the increased levels of $\mathrm{Ca}^{2+}$ and phosphate concentrations in the microenvironment of 


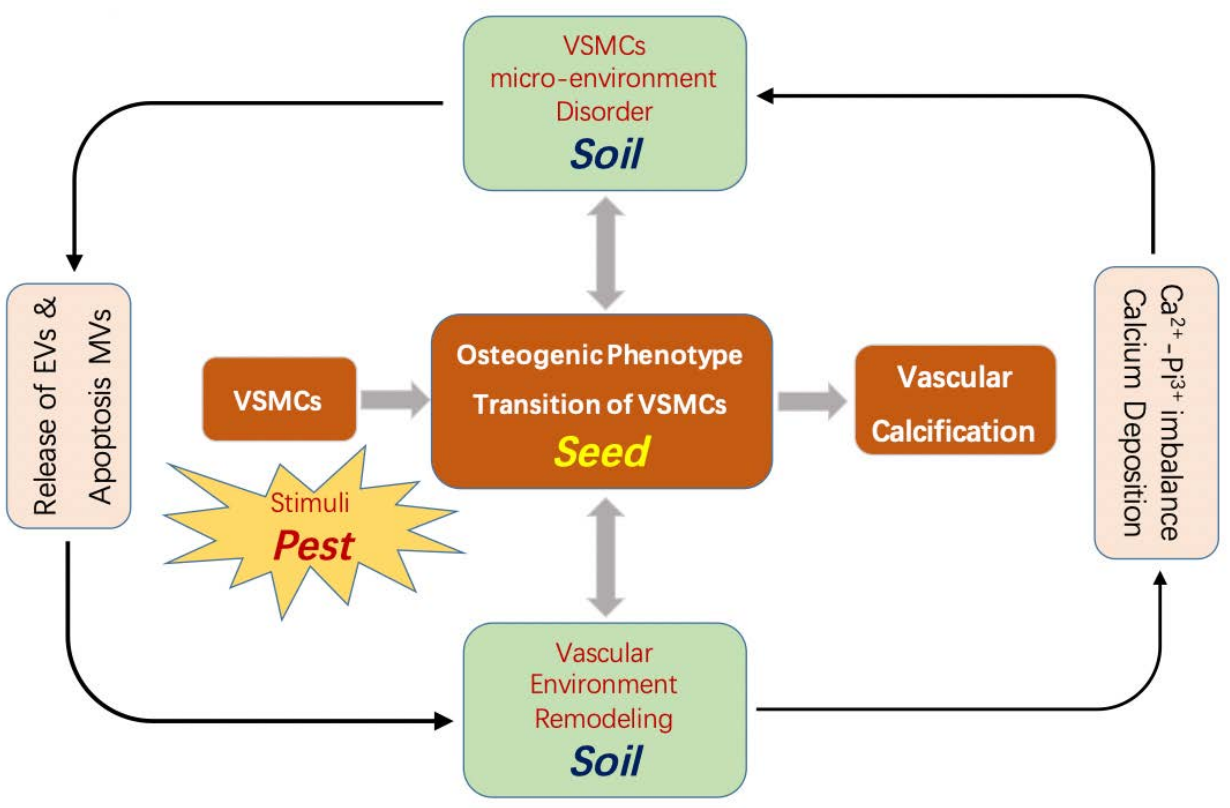

Figure 1: The "Soil-Seed-Pest" mechanism model of VC: alteration and imbalance of VSMCs microenvironment and vascular environment (soil); damage to VSMCs (seed) exposed to calcification environment, especially VSMCs that had transformed into osteogenic phenotype and senescence VSMCs; and various extracellular stimuli (pest) such as ROS damage, inflammation, and hypertension. Alteration in "soil" conditions, "seed" itself defects, and external "pest" stimuli are the three elements that work together and affect each other to form a vicious circle eventually leading to the outcome of VC. VSMC, vascular smooth muscle cell; EV, extracellular vesicle; $\mathrm{MV}$, matrix vesicle.

VSMCs; calcification-promoting factors such as bone morphogenetic protein (BMP) keep increasing; and the level of calcification-inhibiting factors such as OPG were downregulated. VSMCs would release EVs to the matrix, undergo apoptosis, and transform into the osteogenic phenotype. At the same time, with some other factors such as hypertension, vascular remodeling and calcium deposition occur, which changed the vascular environment. This alteration would promote the process of VSMCs phenotype transition and further aggravate the disorder of calcium-phosphorus balance in the VSMCs microenvironment, making the microenvironment more likely to promote the phenotype transition of VSMCs.

VSMCs were the main elements of VC. Senescence VSMCs were much more likely to damage and calcification. In addition, VSMCs that have undergone osteogenic phenotype transition could not recover to the contractile phenotype in a continuous pro-calcification microenvironment, and VSMCs synthesize a large number of osteoblast-related proteins; meanwhile, VSMCs were also under the regulation of calcification signaling and promoting phenotypetransition-related signals, leading to the calcification.

Some extracellular stimuli could change the state of VSMCs, taking the damaged VSMCs into a stress state. In these situations, VSMCs would easily undergo osteogenic phenotype transition if they were exposed to external calcification stimuli. In addition, some extracellular injury could take damage to the "seed," such as ROS damage or inflammatory damage, and also could affect the "soil." For example, hypertension would accelerate vascular microenvironment remodeling and make arteries stiffing, accelerating the calcification process.

Increasing evidence indicates that mitochondrial damage existed in calcified VSMCs, and some research also found that alterations in VSMCs phenotype were related to a different status of mitochondrial metabolism and that calcified blood vessels were often accompanied by mitochondrial damage and dysfunction. Various mechanisms could be involved in mitochondrial dysfunction and lead to calcification of vascular ${ }^{[31-33]}$; therefore, an amount of research has reached a consensus that mitochondria play a key role in the calcification process of VSMCs.

\section{MITOCHONDRIAL FUNCTION AND BEHAVIOR WERE INVOLVED IN THE VC PROCESS}

The mitochondrial electron transport chain (ETC) was the main source of ROS production. ${ }^{[34]}$ ETC consisted of $13 \mathrm{mtDNA}$-encoded respiratory complexes. The Krebs cycle supplied nicotinamide adenine dinucleotide $(\mathrm{NADH})$ and flavin adenine dinucleotide (FAD) to two transmembrane proteins named respiratory complexes I and II, respectively. High-energy electrons moved 
along the respiratory complexes of ETC. At the same time, protons were transported from the matrix to the intermembrane space; the accumulation of protons across the inner membrane provide potential energy for following subsequent reactions. The terminal component of ETC was ATP synthase, which forced inorganic phosphate groups to connect to adenosine diphosphate (ADP) and converted it to ATP. The whole process was defined as oxidative phosphorylation (OXPHOS).${ }^{[3]}$ During the process of transferring electrons, little amounts of electrons would leak from the respiratory complexes of ETC, extraordinarily complexes I and III. ${ }^{[36]}$ The electrons and oxygen molecules combined to form ROS. ${ }^{[36-38]}$ Under a variety of ROS production and clearance mechanisms, ROS levels remained stable at a certain concentration and participate in the regulation of physiological process such as cell proliferation, apoptosis, and $\mathrm{Ca}^{2+}$ storage ${ }^{[39]}$; even $0.01 \mathrm{mmol} / \mathrm{L}$ of hydrogen peroxide $\left(\mathrm{H}_{2} \mathrm{O}_{2}\right)$ could relieve $\mathrm{VC}$-induced ROS production by upregulating nuclear factor related factor-2 (Nrf-2). ${ }^{[40]}$ However, excessive ROS production or insufficient clearance of ROS would give rise to a large accumulation of ROS, resulting in a series of oxidative stress damage caused by the imbalance between oxidative and antioxidant effects in the body.

\section{Mitochondria affect VC directly by oxidative stress damage}

ROS produced by mitochondria further cause oxidative stress damage and eventually cause damage to cells and tissues. This was the primary method that mitochondria participate in the development of various diseases. Some studies have found increased $\mathrm{H}_{2} \mathrm{O}_{2}$ level and mitochondrial injury at the location of the calcified vessel ${ }^{[41]}$; meanwhile in vivo and in vitro experiments confirmed that antioxidant treatment could reduce oxidative stress and improve VC. ${ }^{[42]}$ Mitochondrial ROS might affect calcification by regulating phenotype transition factors of VSMCs such as ALP activity. ${ }^{[43]}$ During oxidative stress, a lot of nicotinamide adenine dinucleotide phosphate (NADPH) oxidases were activated. NADPH oxidase was the main enzyme that generates ROS, and its catalyzed ROS could further promote much more production of ROS from other sources, which led to a large amount of ROS in the body, eventually upregulated the expression of Runx 2 via activating the phosphatidylinositol 3-kinase/protein kinase-B/Runx2 signaling pathway (PI3K/AKT/Runx2 signaling) and promoted the transition of VSMC into osteogenic phenotype. ${ }^{[7,44]}$ And knocking down the level of Runx2 could prevent $\mathrm{H}_{2} \mathrm{O}_{2}$-induced VC, indicating that ROS must depend on the regulation of Runx 2 to cause calcification ${ }^{[7]}$. These results suggested that mitochondrial oxidative stress could cause VC. ${ }^{[45]}$

Pi was one of the substrates for ATP synthesis during
OXPHOS and participated as a key role in regulating the stabilization of mitochondrial membrane potential (MMP) and ROS in mitochondria. ${ }^{[4]}$ Tuyet Thi Nguyen et al. have found that with the transport of Pit-1, the cellular uptake of $\mathrm{Pi}$, which was mediated by Pit-1, causes cytoplasmic alkalization. This change in $\mathrm{pH}$ promoted the transfer of $\mathrm{Pi}$ into the mitochondrial matrix. The increase in Pi uptake level in mitochondrial accelerated the production of ROS. ${ }^{[47]}$ In the Pi-induced VSMCs calcification, it was found that the MMP was gradually decreased and accompanied by a decrease in intracellular ATP level. Oxygen consumption was also decreased at the end stage of VC. However, after treatment with alpha-linolenic acid (ALA), which was known to enhance mitochondrial metabolism and increase ATP production, it was found that the MMP gradually returned to a normal level, ATP production increased and Pi-induced alterations in the mitochondrial structure was reversed. Meanwhile, VC has been significantly rescued. ${ }^{[48]}$ These results indicated that Pi could indeed result in mitochondrial structural damage and dysfunction, further leading to calcification of cells.

ROS was the main "tool" of mitochondria to execute cellular damage, and some studies have reported several potential signaling pathways for how ROS mediates VC. Byon et al. have demonstrated that $\mathrm{H}_{2} \mathrm{O}_{2}$ could significantly activate extracellular regulated protein kinases (ERKs), AKT, and phospholipase C-gamma (PLC $\gamma$ ) signaling pathways. However, neither mitogen-activated protein kinase (MAPK kinase) nor PLC inhibitor could reverse the VC induced by $\mathrm{H}_{2} \mathrm{O}_{2}$, while PI3K/AKT blockers could significantly inhibit $\mathrm{VC}$, indicating that the signaling pathway of PI3K/AKT mediates the role of $\mathrm{H}_{2} \mathrm{O}_{2}$ in regulating VC. ${ }^{[7]}$ High concentration phosphate stimuli promote the increase in mitochondrial ROS by changing the MMP and activating the nuclear factor-kappa B (NF$x \mathrm{~B})$ signal pathway, promoted the VSMCs to transform into osteogenetic phenotype and finally leads to VC. ${ }^{[49]}$ In the beta-glycerophosphate $(\beta-G P)$-induced bovine aorta smooth muscle cells calcification by Zhao et al., they found that mitochondrial ROS activated NF- $x \mathrm{~B}$ signaling and $\mathrm{NF}-x \mathrm{~B}$ subunit $\mathrm{p} 65$ was translocated in nuclear and further transformed cells into osteogenetic phenotype, leading to calcification. Their finding suggested that mitochondrial ROS-NF- $x$ B signaling pathway participates in $\beta$-GPinduced calcification through mitochondrial-to-nuclear signaling interaction. ${ }^{[50]}$ Further research found that NF$x \mathrm{~B}$ signaling pathway was further activated by TNF, which could significantly increase the calcium deposition and phenotype transition of VSMCs. ${ }^{[51]}$ First, NF- $x$ B promoted the expression of $\mathrm{Msx} 2$ and thus promoted the expression of ALP to VSMCs, and second, NF- $x \mathrm{~B}$ promoted the expression of tristetraprolin (TTP), a RNA destabilizing factor, thereby reducing the expression of VC endogenous 
inhibitory factor ankyrin $\mathrm{H}(\mathrm{ANKH})$, and eventually promoting VC process. ${ }^{[9,51]}$ However, at the physiological level of Pi concentration, activation of NF- $x \mathrm{~B}$ signaling pathway could not lead to VSMCs calcification, ${ }^{\left[{ }^{[9} \text { indicating }\right.}$ that NF- $x \mathrm{~B}$ signal's activation was just involved in a part of the regulation process in high concentration phosphateinduced calcification. Some research on the brain and nervous systems suggest that the regulatory effects of ROS on NF- $x \mathrm{~B}$ were two ways, depending on the concentration of ROS. Low levels of ROS could activate NF- $x$ B, but if too much ROS was generated, it could inhibit the activity of $\mathrm{NF}-x \mathrm{~B}$. Activation of NF- $x \mathrm{~B}$ by ROS would have different effects under different conditions of stimuli or issues. ${ }^{[52-54]}$ The process of high-concentration phosphate-induced calcification of VSMCs involved multiple pathways, and there were other approaches to regulation.

In Pi-treated VSMCs, besides mitochondrial structural damage, there was also mitochondrial fission, excessive mitochondria fragmentation, and enhanced expression and phosphorylation of dynamin-related protein 1 (Drp1). Cui et al. used quercetin treatment to reduce mitochondrial fission by inhibiting oxidative stress and inhibiting the expression or phosphorylation of Drp1, thereby blocking phosphate-induced VSMCs apoptosis and calcification. ${ }^{[5]}$ In addition, mitochondrial dysfunction could lead to decreased ATP production; recently, an exome sequencing of elastic fiber indicates multiple sequence variants of solute carrier family 25 member 5 (SLC25A5) gene were a bridge of elastic fiber and ADP-to-ATP ratio and further affected extracellular matrix and upregulated promoting osteogenic factors. ${ }^{[5]}$

\section{Mitochondria indirectly regulate the VC process}

VSMCs were the main cell component of blood vessels, maintaining the tension of vessels and withstanding mechanical shear stress, so it is important for VSMCs to maintain the stability of function. ${ }^{[57-59]}$ Autophagy was a conserved intracellular degradation approach. By forming autophagosomes and fusing with lysosomes, macromolecules and organelles were degraded to maintain cell homeostasis. ${ }^{[60]}$

Autophagy was proved to have the ability to regulate $\mathrm{Ca}^{2+}$ transfer and maintain calcium homeostasis in VSMCs. In a mice model with VSMC-specific autophagy-related gene 7 (Atg7) deletion, autophagy of VSMCs was defective, results in breaking the balance of $\mathrm{Ca}^{2+}$ uptake and release. The voltage-gated $\mathrm{Ca}^{2+}$ channels that promote $\mathrm{Ca}^{2+}$ from extracellular into cells were more sensitive to depolarization, leading to increased intracellular $\mathrm{Ca}^{2+}$ level. ${ }^{[61]}$ Mitochondria could accumulate calcium in an energydependent manner, and mitochondria play a key role in regulating $\mathrm{Ca}^{2+}$ homeostasis in the cell. ${ }^{[62]}$ Excessive intake of $\mathrm{Ca}^{2+}$ by mitochondria would trigger the opening of permeability switching pores and release cytochrome $\mathrm{C}$ into the matrix, which led to apoptosis response. ${ }^{[63]}$ The imbalance of intracellular calcium could directly mediate the calcification of VSMCs. According to the previous research, it was currently believed that calcium regulates VSMC calcification mainly through the formation of mineral cores sites and early-stage events involved in the calcification cascade. On the one hand, calcium in the cells promoted the release of EVs, and on the other hand, calcium could change the intrinsic characteristics of EVs and make it easy for the EVs to form crystalline hydroxyapatite. ${ }^{[64,65]}$

In the recent years, some research has shown that autophagy has a significant inhibition effect on the development of VC. Dai et al. found that high phosphate stimuli could promote the formation of autophagosomes in VSMCs. And after 3-methyladenine (3-MA) treatment, to inhibit the formation of autophagosomes, the calcification would be significantly aggravated. ${ }^{[14]}$ Liu $e t$ al. also found that atorvastatin could inhibit transforming growth factor-beta1 (TGF- $\beta 1$ )-induced VSMCs calcification by inhibiting the $\beta$-catenin signaling pathway, thereby inducing an increase in autophagy levels. ${ }^{[6]]}$ Metformin has been proved to have the ability to induce upregulation of autophagy to recover the state of mitochondrial biogenesis and phosphateinduced damage in VSMCs and block phenotype transition in VSMCs. ${ }^{[3]}$ These results indicated that mitochondrial autophagy was defective in VC. Upregulation of mitophagy might restore mitochondrial function. ${ }^{[67]}$ Highconcentration phosphate could promote the production of mitochondrial superoxide anion and then activate the autophagy of VSMCs. When autophagy was suppressed, the calcium deposition was increased and VSMCs released more MVs and EVs, which contained high ALP activity and accelerated the development of VC. ${ }^{[29]}$

Autophagy happened in mitochondria specially to selectively remove damaged and unnecessary mitochondria were defined as mitophagy. Mitophagy was a protective behavior that maintains mitochondrial homeostasis when mitochondria were under some stimuli such as cellular ROS accumulation, mitochondrial swelling, and $\mathrm{mtDNA}$ damage. ${ }^{[68,}{ }^{69]}$ In the calcified VSMCs induced by lactate, it was found that mitochondrial dysfunction and the mitophagy level were downregulated. However, increased levels of mitophagy, mediated by B-cell lymphoma 2 (Bcl-2)-interacting protein 3 (BNIP3), could reverse mitochondrial disorders and inhibit the VSMCs' phenotype converting to an osteoblastic phenotype. ${ }^{[70]}$ These results suggested that autophagy and mitophagy worked as an anticalcification and anti-oxidative stress factor and maintained the homeostasis of mitochondria and VSMCs. 
Apoptosis was a sort of programmed cell death protocol, defined as cells take place an orderly death, unlike necrocytosis, executed by related genes to keep the microenvironment of the cells to remain stable. ${ }^{[11,72]} \mathrm{At}$ present, we knew there were two main apoptotic pathways: the extrinsic or death receptor pathway and the intrinsic or mitochondrial pathway. However, there was increasing evidence, indicating that the two pathways were interrelated and that molecules in one pathway could affect the other. ${ }^{[73]}$

Therole of apoptosis mechanismin VCwasalso confirmed. ${ }^{[7,75]}$ Apoptosis promoted the bovine VSMCs calcification induced by phosphate. Conversely, the application of apoptosis inhibitors could inhibit the deposition of calcium and cells phenotype transform. Apoptotic bodies released by apoptotic VSMCs were one of the mechanisms that promote VC. ${ }^{[76]}$ In vitro experiments have demonstrated that calcification has a closed linkage to the significantly increased apoptosis level in VSMCs. Calcification could be rescued significantly by decreased release of calcified MVs with the use of apoptosis inhibitors. ${ }^{[15]} \mathrm{H}_{2} \mathrm{O}_{2}$ could induce VSMCs apoptosis level and downregulate Bcl-2 expression significantly, which was an anti-apoptotic protein, suggesting that $\mathrm{H}_{2} \mathrm{O}_{2}$ may induce VSMCs apoptosis in the way of downregulating the expression of anti-apoptosis factors such as Bcl-2. ${ }^{[77]}$

Mitochondria-dependent apoptotic events participated in the process of VC. In some damage conditions such as high phosphate concentration environment, mitochondrial membrane integrity was compromised; mitochondria would release cytochrome $\mathrm{C}$ from the mitochondrial inner membrane space into the cytoplasm. ${ }^{[78]}$ Cytochrome C bound and activated apoptotic protease activating factor-1 (Apaf-1) as well as caspase- 3 and caspase-9, leading to DNA fragmentation and other alterations that cause apoptosis and death. ${ }^{[79]}$ Mitochondria were involved in intrinsic apoptotic regulation. ${ }^{[80,81]} \alpha$-Lipoic acid was a natural antioxidant with antiapoptotic activity; some studies have confirmed $\alpha$-lipoic acid could rescue phosphate-induced $\mathrm{VC}$ and related apoptosis, and further results demonstrated that $\alpha$-lipoic acid could activate growth arrest-specific 6 (Gas6)/Axl/Akt pathway to restore mitochondrial function and inhibit the activity of mitochondria-related apoptosis. ${ }^{[82,83]}$ These defects were associated with apoptotic events, indicated there might be a potential linkage between mitochondrial dysfunction and phosphate-induced apoptosis and calcification.

Apoptosis was related to autophagy. Apoptosis, under calcification stimuli, could be considered a severe consequence of failure of autophagy to re-establish balance for VSMCs. Autophagy could inhibit the apoptotic response by removing damaged mitochondria. ${ }^{[84,85]}$
Autophagy has been shown to be a protective factor of Pi-induced calcification and played a key role in arterial calcification, ${ }^{[29]}$ although, in some tissues, the apoptotic response could be enhanced by autophagy. ${ }^{[86]}$

\section{MITOCHONDRIAL DNA DAMAGE AND VC}

mtDNA was a type of genetic material specific to mitochondria and independent of nuclear DNA (nDNA). Each mitochondrion contained one or more copies of mtDNA, which were located in the mitochondrial matrix near the inner membrane and have a closed doublestranded circular structure. ${ }^{[87]}$

mtDNA was mainly responsible for encoding 13 subunits of the crucial OXPHOS components, ${ }^{[88]}$ including 7 subunits of complex I, 1 subunit of complex III, three subunits of complex IV, and 2 subunits of complex V to assemble the mitochondrial respiratory chain and construct an oxidative phosphorylated respiratory chain and complete electron transfer to produce ATP. ${ }^{[8]}$ Unlike nDNA, mtDNA did not combine with histones, and DNA polymerase gamma (Pol $\gamma$ ) was the only enzyme in mitochondria that was the charge of replication and correction of mtDNA, leading to the DNA damage repair system of mitochondria was relatively simple; therefore, mtDNA had poor stability and was prone to take place mutation or deletion. ${ }^{[0]}$ Mitochondrial diseases were manifested as mitochondrial ETC dysfunction. These diseases were caused by mtDNA with pathogenic mutations or mtDNA synthetic-related proteins encoded by nuclear genes.

\section{Inherited mtDNA damage and VC}

In addition to $\mathrm{mtDNA}$ damage caused by some pathological stimuli, hereditary mtDNA mutations or deletions, common in maternally inherited mitochondrial diseases, may be related to mitochondria distributed predilection, nervous system and muscle tissue were more preference to occur this type of disease, such as chronic progressive external ophthalmoplegia, myoclonic epilepsy, mitochondrial encephalomyopathy, and stroke-like episodes. ${ }^{[1]}$ In this kind of disease, VC occurs relatively rarely, but we could usually find severe calcification in other tissues such as cerebrum. Some cases report that the mutation at position 5513 from $G$ to $A$ of mtDNA led to serving calcification in the basal ganglia ${ }^{[92]}$ and the T-A nucleotide pairs deletion in positions 3271 to 3273 of mtDNA, which was responsible for encoding transfer RNA (tRNA), also led to calcification in patient's cerebrum. ${ }^{\left[{ }^{[3]}\right.}$ The phenomenon of tissue predisposition may be related to the threshold effect of abnormal mtDNA. Pathological changes would occur only when a certain amount of mutated mtDNA reaches 
a threshold. The threshold in the central nervous system was the lowest and the most injured and cardiovascular system relatively has a higher threshold; this characteristic of vascular might be a novel insight to the treatment of mitochondria-related calcification. In patients with these inherited mitochondrial diseases, the mechanism was often the deletion or mutation in some sites of mtDNA that leads to the damage of tRNA or ribosomal (rRNA), which further affected the synthesis of oxidative phosphorylation complexes and eventually led to mitochondrial dysfunction. Therefore, it seemed to be another novel potential-related risk factor of $\mathrm{VC}$.

\section{Pol $\gamma$-related mtDNA damage and VC}

Pol $\gamma$, encoded by the gene POLG, was the only known DNA polymerase that local in mitochondria and takes charge of mtDNA replication and maintenance. ${ }^{[94]}$ Pol $\gamma$ was a type of multifunctional enzyme; the catalytic subunit of Pol $\gamma$ has 5'-3' DNA polymerase activity and 3'-5' exonuclease activity for $\mathrm{mtDNA}$ replication, correction, and proofreading. Therefore, the mutation of POLG or damage of Pol $\gamma$ would cause some mtDNA damage, such as mtDNA mutation, deletion of mtDNA copy number, and even mtDNA depletion. Mutation of POLG has become a major cause of mitochondrial disease. ${ }^{\left[{ }^{95]}\right.} \mathrm{A}$ case reported that a patient with severe neurological disease has a mutation occurred in POLG (c3239G > c; p.Ser1080Thr), and the computed tomography (CT) results of the brain indicated extensive intracranial calcification, which was especially much more concentrated in the dentate nucleus and cerebellar hemispheres. ${ }^{\left[{ }^{[6]}\right.}$ A study reported that POLG-/-ApoE-/-mice had a higher incidence of atherosclerosis compared to POLG+/+ApoE-/- mice; the results suggested that the progress and status of atherosclerosis could be directly accelerated by mtDNA damage, while arteries atherosclerosis, as a progressive disease, would eventually lead to calcification of the arterial intima when the disease progress to the end stage. Therefore, we speculated that POLG-related mtDNA damage could promote the development of vascular diseases and participate in the end-stage calcification process indirectly. ${ }^{[97]}$

Mice with POLG D257A mutation expressing Pol $\gamma$ proofreading defective versions accumulated nearly 2 -fold mtDNA mutations than wild-type mice and show accelerated aging characteristics. ${ }^{[8-100]}$ Accumulation of mutations mtDNA eventually led to mitochondrial dysfunction. Mice with mtDNA mutation exhibited a profound elevated oxidative stress, apoptosis, and decreased ATP-to-ADP ratio or decreased ATP production, which led to mitochondrial dysfunction. ${ }^{[101]}$ Some researches had found that the prevalence of arterial stiffening in $P O L G^{\mathrm{D} 257 \mathrm{~A} / \mathrm{D} 257 \mathrm{~A}}$ mice was higher than that in the wild- type mice group, and the intravascular blood flow had a greater shear force on the vessel walls, and this excessive mechanical pressure was involved in the process of $\mathrm{VC}$, so this phenomenon suggested a potential linkage between POLG and VC. ${ }^{[102]}$ Defects in $\mathrm{mtDNA}$ caused structural damage in the respiratory complexes of ETC and further impaired the function of the complexes; eventually these damages would lead to abnormal OXPHOS and energy deficiency. ${ }^{[103]}$

Taken together, although the mechanism of calcification was not clear, and it was still unclear whether these mtDNA-related VC were the primary phenomenon or a secondary phenomenon, we believed that the occurrence of calcification was related to the damage of mtDNA. Whether it was a genetic defect or an abnormal synthesis of mtDNA, the calcification induced by mtDNA damage affected the formation of the mitochondrial OXPHOS complex, which belonged to the damage in the early stage of OXPHOS synthesis. It was a type of structural damage of ETC that led to OXPHOS dysfunction and was different from external stimuli such as high phosphate.

Mitochondrial-damage-induced calcification was a complex process involving multiple factors (Fig. 2). ROS was a core factor for VSMCs calcification caused by mitochondrial disorders. Mitochondria were directly involved in the calcification process via ROS pathway, especially the phenotype transition of VSMCs. Pol $\gamma$ damage or hereditary mtDNA damage led to abnormal ETC synthesis, or even incomplete structure of ETC, resulting in excessive production of ROS. The first target ROS attacked was mtDNA, which caused damage or deficiency of mtDNA, resulting in a vicious circle. Some external damage, such as high-phosphate environment or NADPH activation, would affect the normal operation of ETC and generate ROS. ROS accumulated in mitochondria could affect the activity of ATP synthase and the release of vesicles or even promote the activation of osteoblasts. In addition, excessive ROS could cause mitochondrial fusion-fission imbalance through the activity of Drp-1, resulting in mitochondrial damage and promoting apoptosis. At the same time, ROS signals could activate the NF- $x \mathrm{~B}$ signaling pathway and the PI3K/AKT/Runx2 pathway to promote osteogenic phenotype transition of VSMCs. Mitochondria indirectly regulated the calcification of VSMCs through autophagy/ mitophagy and apoptosis/endogenous apoptosis. Both apoptosis and autophagy were cell degradation mechanisms. The two behavior interacted with each other and maintained a relative balance. Some stimuli that caused damage to MMP, such as ROS or high phosphate, often caused structural damage to the mitochondria, released cytochrome $\mathrm{C}$ from mitochondria, and activated 


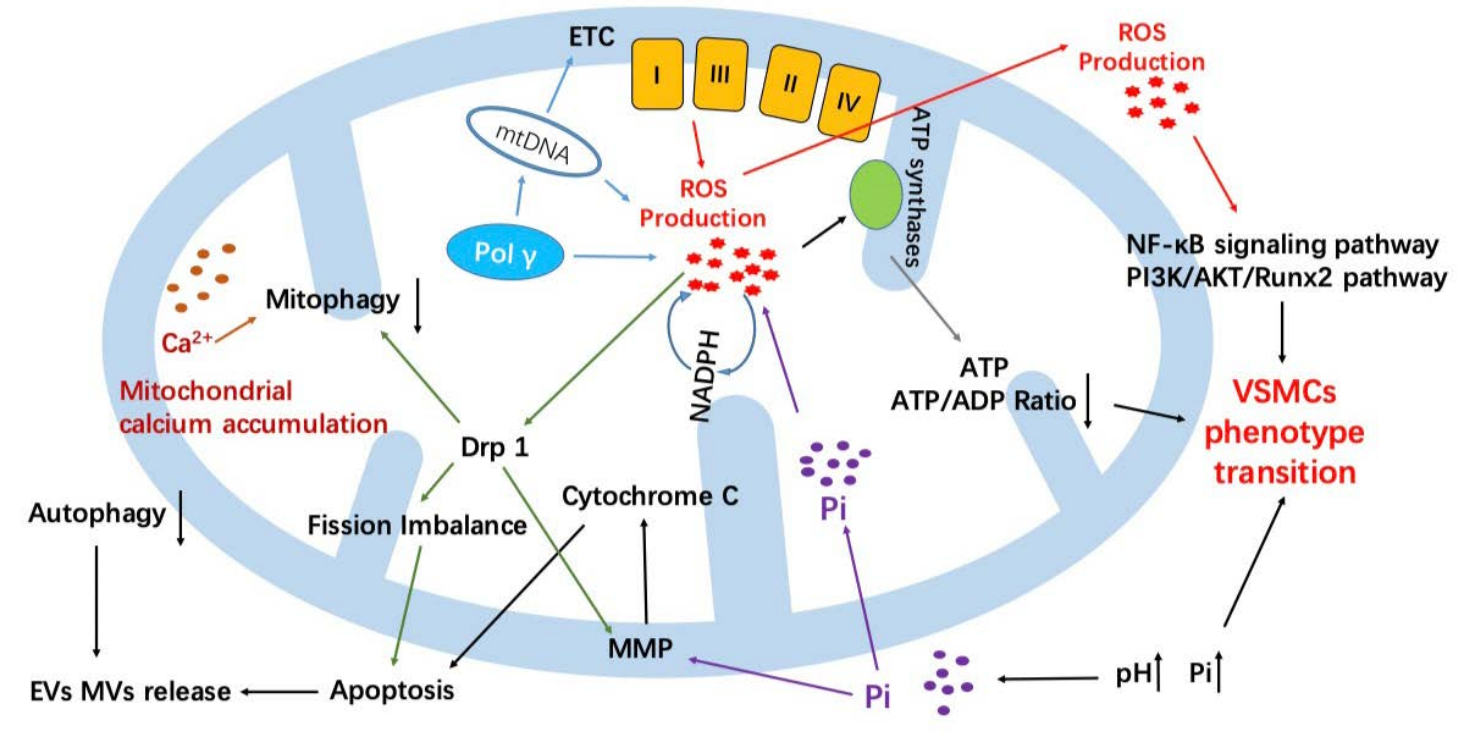

Figure 2: Summary of mitochondrial-damage-induced VC. In total, mitochondria could affect VC in three main ways. Direct damage to mtDNA caused by various of factors could damage the structure and function of ETC, such as mtDNA inherited defects or POLG-related Pol $\gamma$ defects and high-phosphate environment VC such as the high-phosphate environment or due to POLG mutations and eventually lead to VC. Second, mitochondria could also directly participate in the process of VC by oxidative stress injury. ROS is a core factor for VSMCs calcification caused by activating related signaling pathway or breaking the balance of mitochondrial fusion-fission. Finally, mitochondria could indirectly regulate VC process through some biological behavior, such as autophagy/mitophagy and apoptosis; these behaviors could regulate the release of EVs or MVs and further participate in the development of VC. ETC, electron transport chain; mtDNA, mitochondrial DNA; Pol $\gamma$, DNA polymerase gamma; ROS, reactive oxygen species; NADPH, nicotinamide adenine dinucleotide phosphate; Drp1, dynamin-related protein 1; EV, extracellular vesicle; $M V$, matrix vesicle; Pi, inorganic phosphate; ATP, adenosine triphosphate; ADP, adenosine diphosphate; NF- $\varkappa$ B, nuclear factor-kappa B; PI3K, phosphatidylinositol 3-kinase; AKT, protein kinase-B; Runx2, Runt-related transcription factor 2; VSMC, vascular smooth muscle cell; MMP, mitochondrial membrane potential.

endogenous apoptosis, further promoting the release of MVs and leading to calcification. At present, autophagy was considered a protective factor that inhibits the occurrence of calcification and was regulated by many aspects. The large accumulation of $\mathrm{Ca}^{2+}$ in mitochondria led to autophagy damage. And autophagy could reduce endogenous apoptosis to inhibit VC. In addition, there was a close relationship between mitochondria and endoplasmic reticulum (ER). ER, as a calcium bank of VSMCs, takes a great effort in maintaining calcium homeostasis and the process of VC. Endoplasmic reticulum stress (ERS) has received much attention in the recent years, and mitochondrial oxidative stress damage was also one of the causes of ERS; thus ERS may also participate in the calcification process caused by mitochondrial damage.

\section{APPLICATION AND PROSPECT}

For now, there was a little clinical study on mitochondrial function and VC, but some in vivo experiments in model animals have found that some drugs or processes might participate and affect the relationship between mitochondrial function and VC. In vivo experiments in rats have confirmed that resveratrol could improve mitochondrial function and reduce oxidative stress damage, further alleviating the degree of VC. ${ }^{[104]} \mathrm{In}$ the recent years, more attention was focus on exosomes, and studies have found that exosome treatment could also participate in the regulation of mitochondrial function to relieve calcification of VSMCs. ${ }^{[105]}$ Meanwhile, in vivo studies found that Atg7related autophagy could affect the senescence process of POLG mutation mice. ${ }^{[106]}$ In addition, the autophagy process could also maintain the mitochondrial homeostasis to regulate calcification process. ${ }^{[107]}$

With the maturation of gene-editing technology, there were more increasing studies on the root of diseases, so in the future, the regulation of mtDNA stability or even directly affecting POLG might be a potential therapeutic target. Therefore, some measures point to mitochondria might also be a novel insight into VC. Mitochondria were the most common areas where oxidative stress damage occurs, so the intervention in this process should be an effective way to maintain mitochondrial function stability. Mitochondrialtargeted catalase (mCAT) could eliminate excess $\mathrm{H}_{2} \mathrm{O}_{2}$ in mitochondria, maintain the integrity of mitochondrial membrane structure, and regulate mitochondrial-derived apoptosis. A study demonstrated the mitochondria's function suffered from oxidative stress damage in mice overexpressing mCAT was significantly alleviated than wild-type mice. ${ }^{[108]}$ In addition, in the POL $G^{\mathrm{D} 257 \mathrm{~A} / \mathrm{D} 257 \mathrm{~A}}$ mice, which was a mice model of mitochondrial damage, in vivo or in vitro experiments found that mCAT could significantly 
improve the senescence symptoms of POLG $G^{\mathrm{D} 257 \mathrm{~A} / \mathrm{D} 257 \mathrm{~A}}$ mice and the lifespan of POLG $G^{\mathrm{D} 257 \mathrm{~A} / \mathrm{D} 257 \mathrm{~A}} / \mathrm{mCAT}$ mice was longer significantly. ${ }^{[109]}$ Meanwhile, a study indicated that mCAT could also delay the senescence of cerebrovascular. ${ }^{[110]}$ Therefore, taken the above results together, we believed that various methods to improve mitochondria function could regulate the process of VC.

\section{SUMMARY}

Most of the VC was accompanied by mitochondrial dysfunction. Various mechanisms could affect the development of VC through the mitochondrial pathway. These researches provided a new target for the clinical treatment of VC; it was hoped to reduce VMSCs calcification by improving mitochondrial function and further to improve the poor outcome of chronic diseases such as cardiovascular and cerebral diseases and chronic kidney disease.

\section{Source of Foundation}

Natural Science Foundation of China (Grant NO.
81670231)

\section{Conflict of Interest}

The authors declare that there is no conflict of interest.

\section{REFERENCES}

1. Nicoll R, Henein M. Arterial calcification: A new perspective? Int J Cardiol 2017; 228: 11-22.

2. Becs G, Zarjou A, Agarwal A, Kovács KÉ, Becs Á, Nyitrai M, et al. Pharmacological induction of ferritin prevents osteoblastic transformation of smooth muscle cells. J Cell Mol Med 2016; 20: 217-30.

3. Nicoll R, Henein MY. The predictive value of arterial and valvular calcification for mortality and cardiovascular events. Int J Cardiol Heart Vessel 2014; 3: 1-5.

4. Demer LL, Tintut Y. Inflammatory, metabolic, and genetic mechanisms of vascular calcification. Arterioscler Thromb Vasc Biol 2014; 34: 715-23.

5. Yahagi K, Kolodgie FD, Lutter C, Mori H, Romero ME, Finn AV, et al. Pathology of Human Coronary and Carotid Artery Atherosclerosis and Vascular Calcification in Diabetes Mellitus. Arterioscler Thromb Vasc Biol 2017; 37: 191-204.

6. Liberman M, Marti LC. Vascular Calcification Regulation by Exosomes in the Vascular Wall. Adv Exp Med Biol 2017; 998: 151-60.

7. Byon CH, Javed A, Dai Q, Kappes JC, Clemens TL, Darley-Usmar $\mathrm{VM}$, et al. Oxidative stress induces vascular calcification through modulation of the osteogenic transcription factor Runx2 by AKT signaling. J Biol Chem 2008; 283: 15319-27.

8. Lau WL, Festing MH, Giachelli CM. Phosphate and vascular calcification: Emerging role of the sodium-dependent phosphate cotransporter PiT-1. Thromb Haemost 2010; 104: 464-70.

9. Zhao G, Xu MJ, Zhao MM, Dai XY, Kong W, Wilson GM, et al. Activation of nuclear factor-kappa $B$ accelerates vascular calcification by inhibiting ankylosis protein homolog expression. Kidney Int 2012, 82: 34-44.

10. Cui RR, Li SJ, Liu LJ, Yi L, Liang QH, Zhu X, et al. MicroRNA-204 regulates vascular smooth muscle cell calcification in vitro and in vivo. Cardiovasc Res 2012; 96: 320-9.

11. Frauscher B, Kirsch AH, Schabhüttl C, Schweighofer K, Kétszeri M, Pollheimer M, et al. Autophagy Protects From Uremic Vascular Media Calcification. Front Immunol 2018; 9: 1866.
12. Demer LL, Tintut Y. Vascular calcification: pathobiology of a multifaceted disease. Circulation 2008; 117: 2938-48.

13. Kapustin AN, Shanahan CM. Calcium regulation of vascular smooth muscle cell-derived matrix vesicles. Trends Cardiovasc Med 2012; 22: 133-7.

14. Leopold JA. Vascular calcification: Mechanisms of vascular smooth muscle cell calcification. Trends Cardiovasc Med 2015; 25: 267-74.

15. Reynolds JL, Joannides AJ, Skepper JN, McNair R, Schurgers LJ, Proudfoot $\mathrm{D}$, et al. Human vascular smooth muscle cells undergo vesiclemediated calcification in response to changes in extracellular calcium and phosphate concentrations: a potential mechanism for accelerated vascular calcification in ESRD. J Am Soc Nephrol 2004; 15: 2857-67.

16. Chen NX, Moe SM. Vascular calcification: pathophysiology and risk factors. Curr Hypertens Rep 2012; 14: 228-37.

17. Wang TKM, Bolland MJ, van Pelt NC, Horne AM, Mason BH, Ames RW, et al. Relationships between vascular calcification, calcium metabolism, bone density, and fractures. J Bone Miner Res 2010; 25: 2777-85.

18. Zhang C, Zhang K, Huang F, Feng W, Chen J, Zhang H, et al. Exosomes, the message transporters in vascular calcification. J Cell Mol Med 2018; 22: 4024-33.

19. Gomel MA, Lee R, Grande-Allen KJ. Comparing the Role of Mechanical Forces in Vascular and Valvular Calcification Progression. Front Cardiovasc Med 2018; 5: 197.

20. Branchetti E, Poggio P, Sainger R, Shang E, Grau JB, Jackson BM, et al. Oxidative stress modulates vascular smooth muscle cell phenotype via CTGF in thoracic aortic aneurysm. Cardiovasc Res 2013; 100: 316-24.

21. Ruffenach G, Chabot S, Tanguay VF, Courboulin A, Boucherat O, Potus F, et al. Role for Runt-related Transcription Factor 2 in Proliferative and Calcified Vascular Lesions in Pulmonary Arterial Hypertension. Am J Respir Crit Care Med 2016; 194: 1273-85.

22. Wu SY, Yu YR, Cai Y, Jia LX, Wang X, Xiao CS, et al. Endogenous aldosterone is involved in vascular calcification in rat. Exp Biol Med (Maywood) 2012; 237: 31-7.

23. Kieffer P, Robert A, Capdeville-Atkinson C, Atkinson J, LartaudIdjouadiene I. Age-related arterial calcification in rats. Life Sci 2000; 66: 2371-81.

24. Adijiang A, Higuchi $Y$, Nishijima F, Shimizu H, Niwa T: Indoxyl sulfate, a uremic toxin, promotes cell senescence in aorta of hypertensive rats. Biochem Biophys Res Commun 2010; 399: 637-41.

25. Kanemaru K, Seya K, Miki I, Motomura S, Furukawa K-I. Calcification of aortic smooth muscle cells isolated from spontaneously hypertensive rats. J Pharmacol Sci 2008; 106: 280-6.

26. Villa-Bellosta R, Millan A, Sorribas V. Role of calcium-phosphate deposition in vascular smooth muscle cell calcification. Am J Physiol Cell Physiol 2011; 300: C210-20.

27. Masumoto A, Sonou T, Ohya M, Yashiro M, Nakashima Y, Okuda $\mathrm{K}$, et al. Calcium Overload Accelerates Phosphate-Induced Vascular Calcification Via Pit-1, but not the Calcium-Sensing Receptor. J Atheroscler Thromb 2017; 24: 716-24.

28. Shanahan CM, Crouthamel MH, Kapustin A, Giachelli CM. Arterial calcification in chronic kidney disease: key roles for calcium and phosphate. Circ Res 2011; 109: 697-711.

29. Dai XY, Zhao MM, Cai Y, Guan QC, Zhao Y, Guan Y, et al. Phosphateinduced autophagy counteracts vascular calcification by reducing matrix vesicle release. Kidney Int 2013; 83: 1042-51.

30. Giachelli CM. Vascular calcification mechanisms. J Am Soc Nephrol 2004; 15: 2959-64.

31. Ma WQ, Sun XJ, Wang Y, Zhu Y, Han XQ, Liu NF. Restoring mitochondrial biogenesis with metformin attenuates $\beta$-GP-induced phenotypic transformation of VSMCs into an osteogenic phenotype via inhibition of PDK4/oxidative stress-mediated apoptosis. Mol Cell Endocrinol 2019; 479: 39-53.

32. Chiong M, Cartes-Saavedra B, Norambuena-Soto I, Mondaca-Ruff D, Morales PE, García-Miguel M, et al. Mitochondrial metabolism and the control of vascular smooth muscle cell proliferation. Front Cell Dev Biol 2014; 2: 72.

33. Zhang CY, Sun XY, Ouyang JM, Gui BS. Diethyl citrate and sodium citrate reduce the cytotoxic effects of nanosized hydroxyapatite crystals on mouse vascular smooth muscle cells. Int J Nanomedicine 2017; 12: 8511-25.

34. Adam-Vizi V. Production of reactive oxygen species in brain mitochondria: contribution by electron transport chain and nonelectron transport chain sources. Antioxid Redox Signal 2005; 7: 1140-9. 
35. Bartolák-Suki E, Imsirovic J, Nishibori Y, Krishnan R, Suki B. Regulation of Mitochondrial Structure and Dynamics by the Cytoskeleton and Mechanical Factors. Int J Mol Sci 2017; 18: 1812

36. Bleier L, Dröse S. Superoxide generation by complex III: from mechanistic rationales to functional consequences. Biochim Biophys Acta 2013; 1827: 1320-31.

37. Steinbrenner $\mathrm{H}$, Sies $\mathrm{H}$. Protection against reactive oxygen species by selenoproteins. Biochim Biophys Acta 2009; 1790: 1478-85.

38. Kauppila TES, Kauppila JHK, Larsson NG. Mammalian Mitochondria and Aging: An Update. Cell Metab 2017; 25: 57-71.

39. Stowe DF, Camara AKS. Mitochondrial reactive oxygen species production in excitable cells: modulators of mitochondrial and cell function. Antioxid Redox Signal 2009; 11: 1373-414.

40. Zhang W, Li Y, Ding H, Du Y, Wang L. Hydrogen peroxide prevents vascular calcification induced ROS production by regulating Nrf-2 pathway. Ren Fail 2016; 38: 1099-106.

41. Miller JD, Chu Y, Brooks RM, Richenbacher WE, Peña-Silva R, Heistad DD. Dysregulation of antioxidant mechanisms contributes to increased oxidative stress in calcific aortic valvular stenosis in humans. J Am Coll Cardiol 2008; 52: 843-50.

42. Peralta-Ramírez A, Montes de Oca A, Raya AI, Pineda C, López I, Guerrero F, et al. Vitamin E protection of obesity-enhanced vascular calcification in uremic rats. Am J Physiol Renal Physiol 2014; 306: F422-F429.

43. Tang F, Chan E, Lu M, Zhang X, Dai C, Mei M, et al. Calpain-1 Mediated Disorder of Pyrophosphate Metabolism Contributes to Vascular Calcification Induced by oxLDL. PLoS One 2015; 10: e0129128-e0129128.

44. Byon $\mathrm{CH}$, Heath JM, Chen Y. Redox signaling in cardiovascular pathophysiology: A focus on hydrogen peroxide and vascular smooth muscle cells. Redox Biol 2016; 9: 244-53.

45. Bartnicki P, Fijałkowski P, Majczyk M, Błaszczyk J, Banach M, Rysz J. Effect of methoxy polyethylene glycol-epoetin beta on oxidative stress in predialysis patients with chronic kidney disease. Med Sci Monit 2013; 19: 954-9.

46. Selivanov VA, Zeak JA, Roca J, Cascante M, Trucco M, Votyakova TV. The role of external and matrix $\mathrm{pH}$ in mitochondrial reactive oxygen species generation. J Biol Chem 2008; 283: 29292-300.

47. Nguyen TT, Quan X, Xu S, Das R, Cha S-K, Kong ID, et al. Intracellular alkalinization by phosphate uptake via type III sodium-phosphate cotransporter participates in high-phosphate-induced mitochondrial oxidative stress and defective insulin secretion. FASEB J 2016; 30: 3979-88.

48. Kim H, Kim HJ, Lee K, Kim JM, Kim HS, Kim JR, et al. $\alpha$-Lipoic acid attenuates vascular calcification via reversal of mitochondrial function and restoration of Gas6/Axl/Akt survival pathway. J Cell Mol Med 2012; 16: 273-86.

49. Al-Aly Z. Phosphate, oxidative stress, and nuclear factor- $\kappa \mathrm{B}$ activation in vascular calcification. Kidney Int 201; 79: 1044-7.

50. Zhao MM, Xu MJ, Cai Y, Zhao G, Guan Y, Kong W, et al. Mitochondrial reactive oxygen species promote $\mathrm{p} 65$ nuclear translocation mediating high-phosphate-induced vascular calcification in vitro and in vivo. Kidney Int 2011; 79: 1071-9.

51. Lee HL, Woo KM, Ryoo HM, Baek JH. Tumor necrosis factor-alpha increases alkaline phosphatase expression in vascular smooth muscle cells via MSX2 induction. Biochem Biophys Res Commun 2010; 391 : 1087-92.

52. Turillazzi E, Neri M, Cerretani D, Cantatore S, Frati P, Moltoni L, et al. Lipid peroxidation and apoptotic response in rat brain areas induced by long-term administration of nandrolone: the mutual crosstalk between ROS and NF-kB. J Cell Mol Med 2016; 20: 601-12.

53. Mo SJ, Hong J, Chen X, Han F, Ni Y, Zheng Y, et al. VEGF-mediated NF$\kappa \mathrm{B}$ activation protects PC12 cells from damage induced by hypoxia. Neurosci Lett 2016; 610: 54-9.

54. Yang CM, Lin CC, Hsieh HL. High-Glucose-Derived Oxidative Stress-Dependent Heme Oxygenase-1 Expression from Astrocytes Contributes to the Neuronal Apoptosis. Mol Neurobiol 2017; 54: 47083.

55. Cui L, Li Z, Chang X, Cong G, Hao L. Quercetin attenuates vascular calcification by inhibiting oxidative stress and mitochondrial fission. Vascul Pharmacol 2017; 88: 21-9.

56. Boraldi F, Lofaro FD, Romano O, Grilli A, Losi L, Moscarelli P, et al. Exome sequencing and bioinformatic approaches reveals rare sequence variants involved in cell signalling and elastic fibre homeostasis: new evidence in the development of ectopic calcification Cell Signal 2019; 59: 131-40.

57. Cahill PA, Redmond EM. Vascular endothelium - Gatekeeper of vessel health. Atherosclerosis 2016; 248: 97-109.

58. Sawada H, Chen JZ, Wright BC, Sheppard MB, Lu HS, Daugherty A. Heterogeneity of Aortic Smooth Muscle Cells: A Determinant for Regional Characteristics of Thoracic Aortic Aneurysms? J Transl Int Med 2018; 6: 93-6.

59. Bai L, Shyy JYJP. Shear Stress Regulation of Endothelium: A Doubleedged Sword. J Transl Int Med 2018; 6: 58-61.

60. Tooze SA, Dikic I. Autophagy Captures the Nobel Prize. Cell 2016; 167: 1433-5.

61. Michiels CF, Fransen P, De Munck DG, De Meyer GRY, Martinet W. Defective autophagy in vascular smooth muscle cells alters contractility and $\mathrm{Ca}^{2+}$ homeostasis in mice. Am J Physiol Heart Circ Physiol 2015; 308: H557-67.

62. Glancy B, Balaban RS. Role of mitochondrial $\mathrm{Ca} 2+$ in the regulation of cellular energetics. Biochemistry 2012; 51: 2959-73.

63. Izzo V, Bravo-San Pedro JM, Sica V, Kroemer G, Galluzzi L. Mitochondrial Permeability Transition: New Findings and Persisting Uncertainties. Trends Cell Biol 2016; 26: 655-67.

64. De Broe M. Calcium regulates key components of vascular smooth muscle cell- derived matrix vesicles to enhance mineralization. Kidney Int 2011; 80: 791-2.

65. Giovannucci E, Liu Y, Hollis BW, Rimm EB. 25-hydroxyvitamin D and risk of myocardial infarction in men: a prospective study. Arch Intern Med 2008; 168: 1174-80.

66. Liu D, Cui W, Liu B, Hu H, Liu J, Xie R, et al. Atorvastatin protects vascular smooth muscle cells from TGF- $\beta 1$-stimulated calcification by inducing autophagy via suppression of the $\beta$-catenin pathway. Cell Physiol Biochem 2014; 33: 129-41.

67. Phadwal K, Feng D, Zhu D, MacRae VE. Autophagy as a novel therapeutic target in vascular calcification. Pharmacol Ther 2020; 206: 107430.

68. Saito T, Sadoshima J. Molecular mechanisms of mitochondrial autophagy/mitophagy in the heart. Circ Res 2015; 116: 1477-90.

69. Liu L, Sakakibara K, Chen Q, Okamoto K. Receptor-mediated mitophagy in yeast and mammalian systems. Cell Res 2014; 24: 787-95.

70. Zhu Y, Ji JJ, Yang R, Han XQ, Sun XJ, Ma WQ, et al. Lactate accelerates calcification in VSMCs through suppression of BNIP3-mediated mitophagy. Cell Signal 2019; 58: 53-64.

71. D'Arcy MS. Cell death: a review of the major forms of apoptosis, necrosis and autophagy. Cell Biol Int 2019; 43: 582-92.

72. Kerr JF, Wyllie AH, Currie AR. Apoptosis: a basic biological phenomenon with wide-ranging implications in tissue kinetics. Br J Cancer 1972; 26: 239-57.

73. Igney FH, Krammer PH. Death and anti-death: tumour resistance to apoptosis. Nat Rev Cancer 2002; 2: 277-88.

74. Shroff RC, McNair R, Figg N, Skepper JN, Schurgers L, Gupta A, et al. Dialysis accelerates medial vascular calcification in part by triggering smooth muscle cell apoptosis. Circulation 2008; 118: 1748-57.

75. Lu Y, Bian Y, Wang Y, Bai R, Wang J, Xiao C. Globular adiponectin reduces vascular calcification via inhibition of ER-stress-mediated smooth muscle cell apoptosis. Int J Clin Exp Pathol 2015; 8: 2545-54.

76. Proudfoot D, Skepper JN, Hegyi L, Bennett MR, Shanahan CM, Weissberg PL. Apoptosis regulates human vascular calcification in vitro: evidence for initiation of vascular calcification by apoptotic bodies. Circ Res 2000; 87: 1055-62.

77. Li PF, Dietz R, von Harsdorf R. Differential effect of hydrogen peroxide and superoxide anion on apoptosis and proliferation of vascular smooth muscle cells. Circulation 1997; 96: 3602-3609.

78. Saelens X, Festjens N, Vande Walle L, van Gurp M, van Loo G, Vandenabeele P. Toxic proteins released from mitochondria in cell death. Oncogene 2004; 23: 2861-74.

79. Hill MM, Adrain C, Duriez PJ, Creagh EM, Martin SJ. Analysis of the composition, assembly kinetics and activity of native Apaf-1 apoptosomes. EMBO J 2004; 23: 2134-45.

80. Liu XR, Li T, Cao L, Yu YY, Chen LL, Fan XH, et al. Dexmedetomidine attenuates $\mathrm{H} 2 \mathrm{O} 2$-induced neonatal rat cardiomyocytes apoptosis through mitochondria- and ER-medicated oxidative stress pathways. Mol Med Rep 2018; 17: 7258-64.

81. Leem J, Lee IK. Mechanisms of Vascular Calcification: The Pivotal Role of Pyruvate Dehydrogenase Kinase 4. Endocrinol Metab (Seoul) 2016; 31: 52-61. 
82. Chen S, Liu G, Long M, Zou H, Cui H. Alpha lipoic acid attenuates cadmium-induced nephrotoxicity via the mitochondrial apoptotic pathways in rat. J Inorg Biochem 2018; 184: 19-26.

83. Son BK, Kozaki K, Iijima K, Eto M, Nakano T, Akishita M, et al. Gas6/ Axl-PI3K/Akt pathway plays a central role in the effect of statins on inorganic phosphate-induced calcification of vascular smooth muscle cells. Eur J Pharmacol 2007; 556: 1-8.

84. Levine B, Klionsky DJ. Development by self-digestion: molecular mechanisms and biological functions of autophagy. Dev Cell 2004; 6: 463-477.

85. Kim KW, Hwang M, Moretti L, Jaboin JJ, Cha YI, Lu B. Autophagy upregulation by inhibitors of caspase- 3 and mTOR enhances radiotherapy in a mouse model of lung cancer. Autophagy 2008; 4: 659-668.

86. Green DR, Galluzzi L, Kroemer G. Mitochondria and the autophagyinflammation-cell death axis in organismal aging. Science 2011; 333: 1109-12.

87. Mishra P, Chan DC. Mitochondrial dynamics and inheritance during cell division, development and disease. Nat Rev Mol Cell Biol 2014; 15: 634-46.

88. Stewart JB, Chinnery PF. The dynamics of mitochondrial DNA heteroplasmy: implications for human health and disease. Nat Rev Genet 2015; 16: 530-42.

89. Inoue JG, Miya M, Tsukamoto K, Nishida M. Complete mitochondrial DNA sequence of Conger myriaster (Teleostei: Anguilliformes): novel gene order for vertebrate mitochondrial genomes and the phylogenetic implications for anguilliform families. J Mol Evol 2001; 52: 311-20.

90. de Souza-Pinto NC, Mason PA, Hashiguchi K, Weissman L, Tian J, Guay D, et al. Novel DNA mismatch-repair activity involving YB-1 in human mitochondria. DNA Repair (Amst) 2009; 8: 704-19.

91. Khandwala K, Ahmed A, Sheikh T. MELAS: A Complex and Challenging Diagnosis. J Coll Physicians Surg Pak 2018; 28: S46-8

92. Cardaioli E, Mignarri A, Cantisani TA, Malandrini A, Nesti C, Rubegni A, et al. Myoclonus epilepsy, retinitis pigmentosa, leukoencephalopathy and cerebral calcifications associated with a novel m.5513G>A mutation in the MT-TW gene. Biochem Biophys Res Commun 2018; 500: 158-62.

93. Shoffner JM, Bialer MG, Pavlakis SG, Lott M, Kaufman A, Dixon J, et al. Mitochondrial encephalomyopathy associated with a single nucleotide pair deletion in the mitochondrial tRNALeu(UUR) gene. Neurology 1995; 45: 286-92.

94. Copeland WC, Longley MJ. DNA polymerase gamma in mitochondrial DNA replication and repair. ScientificWorldJournal 2003; 3: 34-44.

95. Chinnery PF. Mitochondrial Disorders Overview. In: GeneReviews( $\left(\AA^{2}\right)$. edn. Edited by Adam MP, Ardinger HH, Pagon RA, Wallace SE, Bean LJH, Stephens K, Amemiya A. Seattle (WA): University of Washington, Seattle; 1993.

96. Sidiropoulos C, Moro E, Lang AE. Extensive intracranial calcifications in a patient with a novel polymerase $\gamma-1$ mutation. Neurology 2013; 81: 197-8.
97. Yu E, Calvert PA, Mercer JR, Harrison J, Baker L, Figg NL, et al. Mitochondrial DNA damage can promote atherosclerosis independently of reactive oxygen species through effects on smooth muscle cells and monocytes and correlates with higher-risk plaques in humans. Circulation 2013; 128: 702-12.

98. Kujoth GC, Bradshaw PC, Haroon S, Prolla TA. The role of mitochondrial DNA mutations in mammalian aging. PLoS Genet 2007; 3: e24.

99. Williams SL, Huang J, Edwards YJK, Ulloa RH, Dillon LM, Prolla TA, et al. The mtDNA mutation spectrum of the progeroid Polg mutator mouse includes abundant control region multimers. Cell Metab 2010 12: $675-82$.

100. Trifunovic A, Wredenberg A, Falkenberg M, Spelbrink JN, Rovio AT, Bruder CE, et al. Premature ageing in mice expressing defective mitochondrial DNA polymerase. Nature 2004, 429: 417-23.

101. Safdar A, Annis S, Kraytsberg Y, Laverack C, Saleem A, Popadin K, et al. Amelioration of premature aging in mtDNA mutator mouse by exercise: the interplay of oxidative stress, PGC- $1 \alpha$, p53, and DNA damage. A hypothesis. Curr Opin Genet Dev 2016; 38: 127-32.

102. Golob MJ, Tian L, Wang Z, Zimmerman TA, Caneba CA, et al. Mitochondria DNA mutations cause sex-dependent development of hypertension and alterations in cardiovascular function. J Biomech 2015; 48: 405-12.

103. El-Hattab AW, Craigen WJ, Scaglia F: Mitochondrial DNA maintenance defects. Biochim Biophys Acta Mol Basis Dis 2017; 1863: 1539-55.

104. Zhang P, Li Y, Du Y, Li G, Wang L, Zhou F. Resveratrol Ameliorated Vascular Calcification by Regulating Sirt-1 and Nrf2. Transplant Proc 2016; 48: 3378-86.

105. Li S, Zhan JK, Wang YJ, Lin X, Zhong JY, Wang Y, et al. Exosomes from hyperglycemia-stimulated vascular endothelial cells contain versican that regulate calcification/senescence in vascular smooth muscle cells. Cell Biosci 2019; 9: 1

106. Li-Harms X, Milasta S, Lynch J, Wright C, Joshi A, Iyengar R, et al. Mito-protective autophagy is impaired in erythroid cells of aged mtDNA-mutator mice. Blood 2015; 125: 162-74.

107. Phadwal K, Feng D, Zhu D, MacRae VE. Autophagy as a novel therapeutic target in vascular calcification. Pharmacol Ther 2020; 206 107430.

108. Schriner SE, Linford NJ, Martin GM, Treuting P, Ogburn CE, Emond $\mathrm{M}$, et al. Extension of murine life span by overexpression of catalase targeted to mitochondria. Science 2005; 308: 1909-11.

109. Dai DF, Chen T, Wanagat J, Laflamme M, Marcinek DJ, Emond MJ, et al. Age-dependent cardiomyopathy in mitochondrial mutator mice is attenuated by overexpression of catalase targeted to mitochondria. Aging Cell 2010; 9: 536-44.

110. Csiszar A, Yabluchanskiy A, Ungvari A, Ungvari Z, Tarantini S. Overexpression of catalase targeted to mitochondria improves neurovascular coupling responses in aged mice. Geroscience 2019; 41: 609-17.

How to cite this article: Wang $P$, Zhang N, Wu B, Wu S, Zhang $Y$, Sun $\mathrm{Y}$. The role of mitochondria in vascular calcification. Transl Intern Med 2020; 8: 80-90. 